\title{
Innovative solution for climbing and cleaning on smooth surfaces
}

\author{
Guido BELFORTE, Giuliana MATTIAZZO, and Roberto GRASSI \\ Department of Mechanics \\ Technical University "Politecnico di Torino" \\ Corso Duca degli Abruzzi 24-10129-Torino-Italy \\ E-mail: roberto.grassi@polito.it
}

\begin{abstract}
A new generation of cleaning robots for domotic applications based on all-pneumatic technology is on study; the project deals with three main fields: motion, cleaning and drying. Referring to motion, an innovative design using pneumatic cylinders as structural elements is being tested; the implementation of a control made with digital valves permits to reduce costs, decrease setup times and leads to a unit which can be easily reconfigured for various applications. A recently developed series of vacuum cups allows the unit to stick itself on surfaces even if dirty or wet; innovative design and materials were studied to guarantee a firm grip in extreme conditions such as metal or glass covered by lubricating fluids. The structure used for motion system and the solutions for control the unit are shown. The cleaning system is based on pneumatic motor driving of rotating brushes with water injection between them. The drying system is based on fluidic concepts and driven by compressed air; studies about Coanda effect were carried on trying to extract water directly from wet surface without contact. Computational Fluid Dynamic simulations and experimental tests were carried on to validate the models; comparative analysis about performances and fluid consumption are here presented.
\end{abstract}

\section{KEY WORDS}

Cleaning, Climbing robot, Pneumatics, Vacuum cups, Window

\section{INTRODUCTION}

The study and production of robots for domotic application is a relatively recent research field. This kind of robot is actually in continuous development. Huge surface cleaning, and even glass windows or building walls is on study in industrial fields with very different characteristics and innovations. The robot presented in this paper, developed at Politecnico di Torino, deals with a field of application not yet studied: small sized windows cleaning in domestic environment. Such a prototype must present some particular aspect that deal with indoor applications; first of all low cost and limited complexity. For that reason the robot was built with the only degrees of freedom really essential and with pneumatic technology. Moreover a cleaning unit must have the smallest interference with the workspace; then recovering of washing fluid is essential. The most important study of this prototype is about an innovative drying unit capable to collect detergent and 
avoid spoilt. The unit uses compressed air. By means of Coanda effect it generates an air stream on a curved surface close to the window; washing fluid is then extracted directly from the glass and conveyed for further recycling.

\section{STATE OF THE ART IN VERTICAL SURFACE CLEANING}

A huge amount of robot is used to transport tools and operate in remote areas [8] [9]. The state of the art in windows cleaning consists mainly in applications over huge surfaces [4] [5] [10].

An innovative robot, developed for cleaning vertical walls and smooth surfaces in general, comes from Institute for Problem in Mechanics of Russian Science Academy [1] ; it consists in a transportation system on which can be mounted several technological modules. The transporter is a round shaped carter with a wheeled platform inside; by mean of electric motors the wheels allow it to move in all directions. The main body is similar to a turtle and an electric fan provide vacuum inside of it; along the perimeter, a gasket permits to hold inner pressure preventing air (vacuum) losses and to slip on the surface reducing friction. Washing fluid is injected near the cleaning unit from a nozzle connected to a pump.

The Institute for Material Sciences, university of Hannover and Schmalz, a German company, had developed a small robot called Hydra [2] that can climb on vertical surfaces; so it may be useful for cleaning applications. Four suction cups allows the unit to stick on the surface; the motion is demanded to pneumatic actuators with polymer made pneumatic cylinders. Hydra can overcome obstacles less than $40 \mathrm{~mm}$ height; cleaning modules can be connected to the main body.

Another interesting solution is Oktoput, developed to clean huge glass surfaces onboard cruise ships; it is made of two structures, relatively translating, and can travel in all directions. Its mass is approx $140 \mathrm{~kg}$ and its length is about $1 \mathrm{~m}$; the main power source is electric, carried by a $220 \mathrm{~V}$ cable. It can move at an average speed of $1 \mathrm{~m} / \mathrm{min}$ carrying an efficient cleaning unit based on rotating brushes; the grip is assured by eight suction cups of approx $30 \mathrm{~cm}$ diameter each.

The robot developed at the Technical University of Turin is based, as the others, on vacuum cups, but its main characteristics are an extremely simple motion system and an innovative cleaning unit; it can recycle the cleaning fluid with a vacuum recovery unit based on Coanda Effect.

\section{STRUCTURE OF THE ROBOT}

The robot workspace will be a window, so in order to define an example of structure it was simplified as a vertical, rectangular shaped, surface approx 1 by 1.5 meters wide; the unit will have to describe the whole space during operation following a defined path. The active degrees of freedom are limited to two, moreover a self aligning system keeps the robot in vertical position; so the motion system allows the robot to climb vertically and translate side by side. Then the self alignment equipment provides a further passive degree of freedom. The cleaning operation does not require particular displacement precision, so a step motion can give enough mobility combined with a simple structure and control; for these reason a Cartesian structure with pneumatic linear actuators was designed. In figure 1 the kinematics is shown: by extending and retracting legs the robot is able to move in both directions step by step.

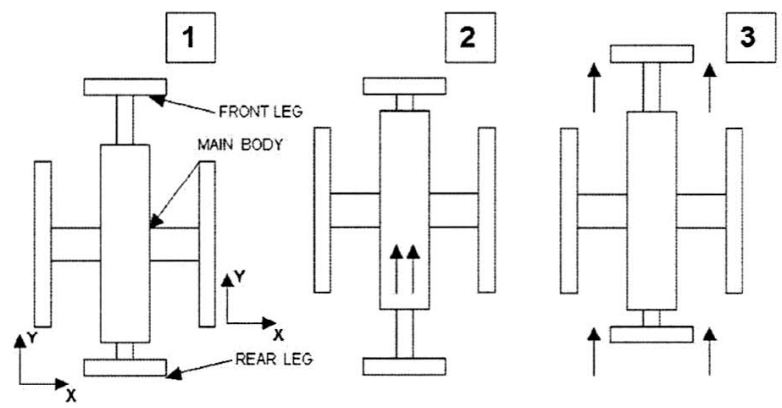

Figure 1: Motion sequence for $\mathrm{Y}$ direction, alternative translation of body and legs allows climbing

The march direction need to be held parallel to the external structure of the window so the easier solution is to suspend the robot like a pendulum: by rotating the body around the upper cylinder it is able to gain a vertical displacement even if it is hanged to the surface in a wrong way. The first group of vacuum cups is mounted on an I shaped frame with an hollow arc of circle, shown in figure 2 , that acts as a circular guide; it permits relative rotation between frame and robot body of approx 10 degrees.

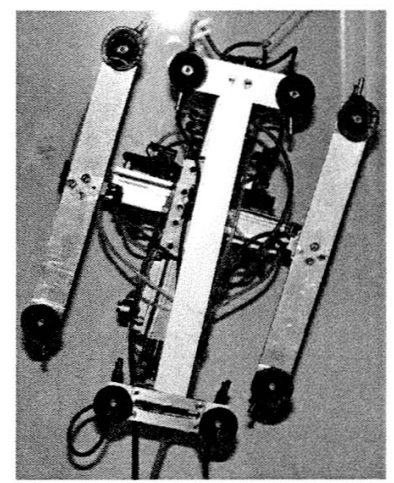

Figure 2: The robot on a Plexiglas window, rear view that shows the self alignment in action. 
The main body consists in four pneumatic cylinders, mounted at a 90 degrees angle, that allows horizontal $X$ and vertical Y motion; the four cylinders are integrated on a central rectangular shaped structure in aluminum. In that way, by using cylinders as structural elements, this compact very light structure can be used to mount cleaning elements or water nozzles; pneumatic actuation allow to fully integrate motors (cylinders) in the whole system in a perfect synergy between actuation and structural elements. Moreover pneumatics guarantee a good reliability with relatively less maintenance and is very cheap; for these reason it was chosen as the best technology for the application. The motion unit can be completed with different implements, so the available fields of operation are extended to all the situations in which transportation of tools might be required; for this goal the structure presents free spaces in the whole body and a maxim payload of approx $50 \%$ of total mass.

In figure 3 the whole robot is shown during a test on a plastic surface. The side legs are visible during extension; on every suction cup there is a pneumatic ejector that generates vacuum.

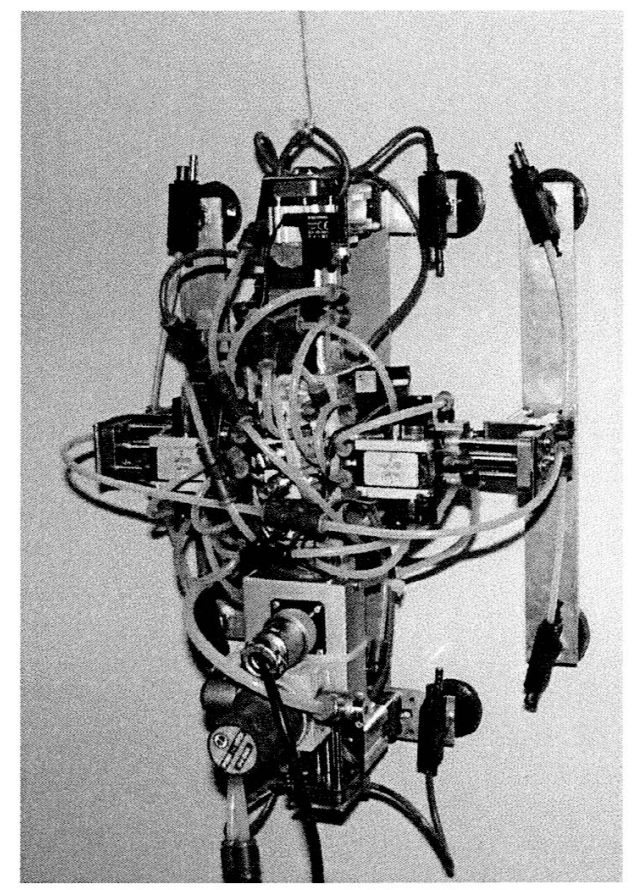

Figure 3: The motion system during a test on a vertical plastic surface.

In order to move the robot along a vertical glass surface, the grip must be firmly and safely assured. Variable forces tend to disengage the unit: as robot mass, that is approx $3 \mathrm{~kg}$; payload due to tools and supply cables; external reaction forces due to particular working. The grip unit consists in eight suction cups.

To assure a correct engage, a wide range of suction cups was analyzed in order to find the best solution. The working environment is the main factor that influences suction cup material: the cup will be working in an extremely wet condition, water and detergent fluid will affect grip performances and weariness resistance. An eventual slip may cause vacuum losses and the whole grip system might become ineffective. The most suitable cup for our application is made in polyurethane with oversized gasket and a diameter of $35 \mathrm{~mm}$. These particular cups do not leave impressions even on extremely smooth surfaces, so during operation the robot will not leave any sign.

\section{COANDA EFFECT DRYER}

The robot was designed to wash and clean glass surfaces, so it must be able to leave the surface clean and dry; moreover the indoor application requires less interactions with the environment. Using brushes and water may cause spoilt of fluid and such event does not happen; for this goal a system able to collect all used water and eventually recycle it was studied. The application of Coanda effect leads to a unit based on a surface, like an aircraft wing, on which an high speed air stream can stick and therefore be driven in a given direction; [7] the air stream, running close to the glass surface, extract water directly and launches it in a direction expected to be perpendicular. Due to the robot structure it can move on the surface in two direction, so the dryer must be able to operate according to the motion; in order to keep the surface clean the drying system will be designed as a rectangular line around the main body. Every side of this rectangle is made of a single drying element; in that way the correct drying in every direction is granted.

In order to design the unit a numerical analysis was carried on. Given its workspace, the dryer is assumed to be a wing shaped element; so a circular hollow body was designed, with a line shaped outlet that permits to drive air from inside to the external surface.

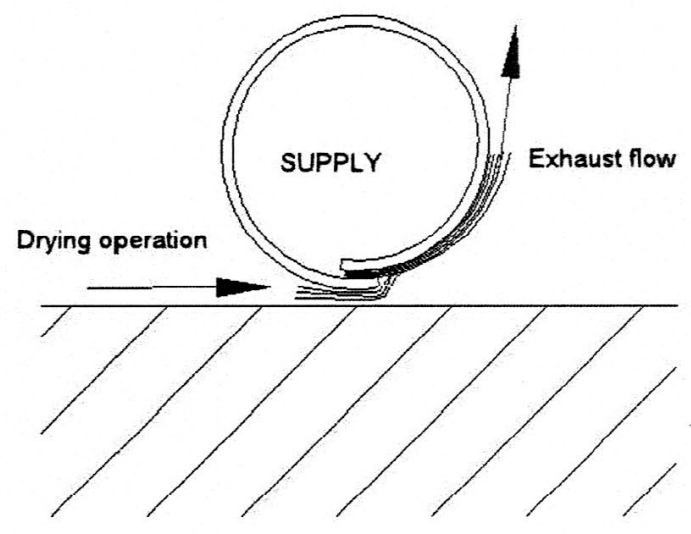

Figure 4: Section of the first prototype. 
After having defined a profile section, the volume to be studied is obtained from. Given the solid geometry the mesh is constructed with particular care; therefore the simulation has been carried out in three dimensions, since some $2 \mathrm{D}$ tests, limited to the profile section have given poor results. The volume object of simulation spreads around the unit. The program simulates fluid behavior in a cylinder $25 \mathrm{~mm}$ diameter around the tube; beyond this distance the effects due to the wall are negligible. In figure 5 the section geometry with linear dimensions is shown.

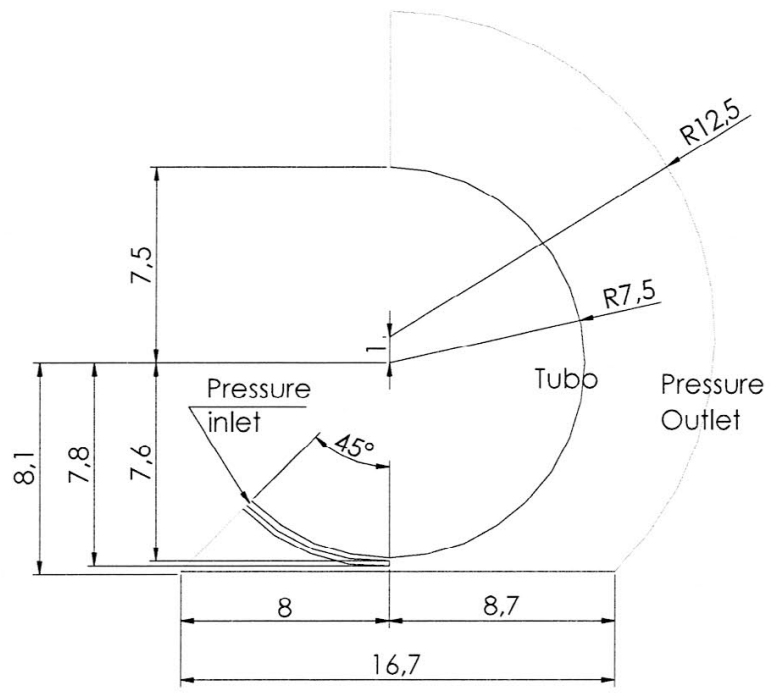

Figure 5:Geometry of the model.

The section, named Pressure Inlet is the system input. The program sets up the pressure in these points as a contour condition; that will be called supply pressure. The mass flow in that particular section will be called supply air flow and is the effective fluid consumption of the object. The section has subsequently been extruded to obtain a profile of $35 \mathrm{~mm}$ length. In order to better evaluate sensitive areas, such as the external curved surface, the mesh has a variable size and is more accurate near the surface. Proceeding that way may be useful in every area in which velocity gradient is supposed to be higher; so the calculation is more accurate only where required and in less important areas the separation is wider. The picture of figure 6 shows a trace of the mesh; in the lowest part there is a line that in solid model represents a wall. To be noticed a more accurate grid between wall and dryer, in that way the interaction between dryer and glass surface will be analyzed with particular care to the distance.

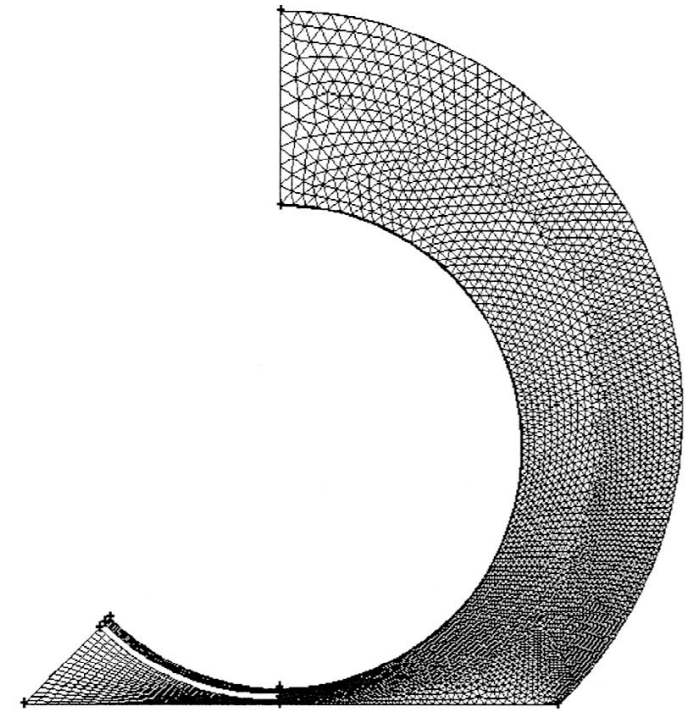

Figure 6: Variable sized mesh.

This model was simulated with pressure supply levels between 0.1 and 0.8 bar; increasing the pressure a first evaluation of air speed is obtained. From these results it is clear that with supply pressure above 0.4 bar the flow has a great Reynolds number and becomes turbulent. In that case the viscous model $\mathrm{K}$-epsilon is used. The results can be represented by graphics. For example air velocity may be represented in a $2 \mathrm{D}$ graphic along a section, as shown in figure 7: in that case the pressure supply is set to 0.4 bar and the mass flow rate is about $220 \mathrm{~g} / \mathrm{min}$.
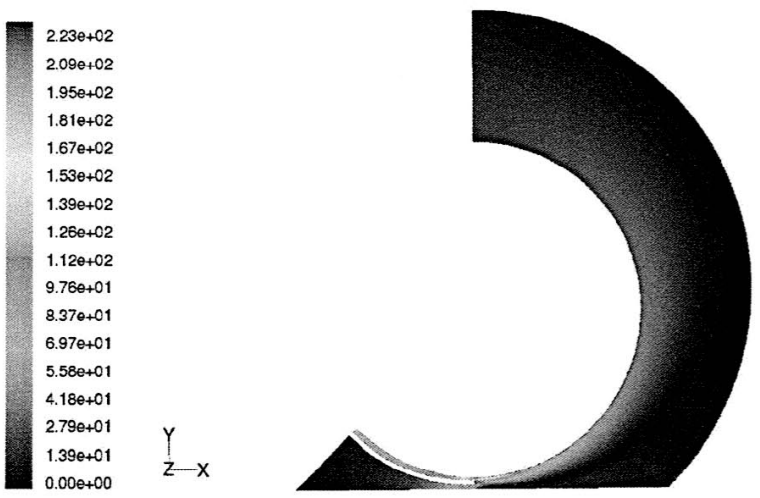

Contours of Velocity Magnitude (m/s) (Time $=2.0000 e-02)$ Sep 22, 2004
FLUENT 6.0 (3d, dp, segregated, ske, unsteady)

Fig. 7 : 2D velocity magnitude plot at supply pressure of 0.4 bar. 
From the figure it can be noticed that in the area next to the wall the velocity presents a maximum; so in these area the airflow is effectively stuck on the surface according to Coanda's theory. Moreover downside of the supply area, between glass surface and the lowest part of the dryer, the flow is particularly intense with an airspeed of $90 \mathrm{~m} / \mathrm{s}$. This is the most interesting aspect because in this area the water extraction effect is generated. After having analyzed the obtained results it was possible to define a useful profile. A good combination between tube diameter ad inlet thickness was defined in order to guarantee an high airspeed.

The theoretical profile was subsequently realized and a first prototype was obtained for experimental tests. In order to validate the model the supply air consumption was evaluated, this is an important characteristic that deals with energy requirements of the system. For this proposal a series of simulations was conducted with variable levels of supply pressure and theoretical data about air consumption were obtained. The air consumption of the prototype is evaluated by giving it a defined supply pressure, then measuring the mass flow rate with a ROTA flow meter at different level of pressure. In figure 8 experimental data of air consumption, in $\mathrm{g} / \mathrm{min}$, are compared with simulation.

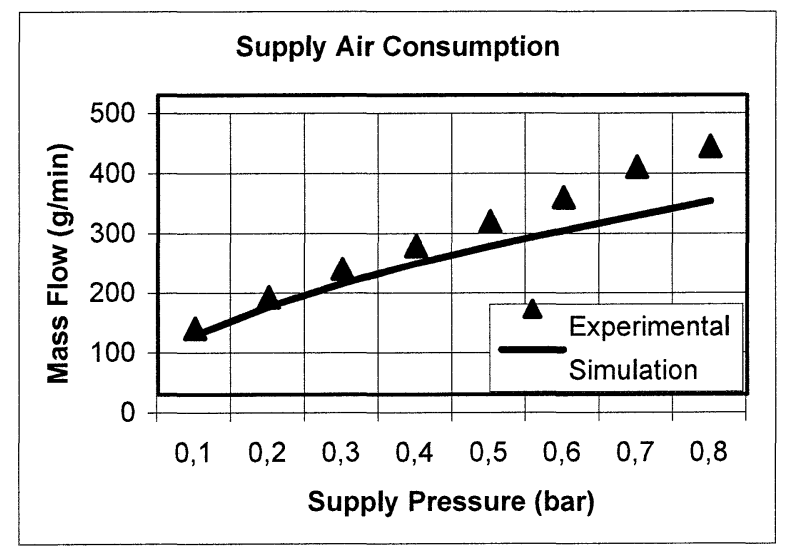

Fig.8 : Air consumption vs supply pressure, experimental data compared with simulation

As noticeable from the figure, experimental data are slightly higher than simulated. The difference between these data increases with supply pressure. This effect is probably caused by the enlargement of inlet section; it is due to the strain of the tube subjected to pressure.

The prototype was subsequently integrated onboard and preliminary evaluation tests were conducted.

The first results are encouraging; the robot climbs on an extremely wet surface and the drying unit extract water leaving glass properly clean and dry.

\section{CONCLUSIONS}

A prototype of a working robot was designed and tested. A Coanda dryer was produced with satisfactory results. Further activities will be carried on to improve the drying capability. In particular a deeper analysis of this application of Coanda effect is required, regarding the geometry, with the goal of reducing supply air consumption maintaining the drying skills.

\section{REFERENCES}

1. V. Gradetsky, M. Rachkov, S. Kalinichenko, E. Semenov : Service robot for cleaning vertical surfaces. IARP Workshop, Genova, October, 1997, pp. 120-126.

2. Un Robot "Scalatore" Rivista di Meccanica $\mathrm{N}^{\circ}$ 1074 Maggio 1995, p.80de

3. Nishi A. : Development of Wall-climbing Robots, Computers Elect. Engignering Volume 22, pp. 123-149, 1996

4. Elkmann N., Felsch T., Sack M., Horting J.: Innovative service Robot for façade cleaning of Difficult-to-Access areas, Proc. 2002 IEEE/RSJ International Conference on Intelligent Robots and Systems

5. Schraft R.D., Simons F.: Facade and window climbing robots for cleaning and inspection, CLAWAR Newsletter, Issue 11 November 2003 pp. 12-16

6. Carpenter P. W., Green P.N.: The aero acoustic and aerodynamics of High-speed Coanda devices, part 1: conventional arrangement of exit nozzle and surface, Journal of Sound And Vibration (1997), pp. 777-801

7. www.jetfan.com

8. S.Yokota, H. Kobayashi, K. Kabawata: Development of a mobile system using leg-type crawlers for rough terrains, CLAWAR Newsletter 2002 p. 899.

9. M.Armada, P.Gonzales de Santo, M.Prieto : Climbing and walking robot for the petrolchemical industry and for underwater applications, CLAWAR Newsletter 2002 p. 939.

10. O.Derriche, K.Kouiss : A cleaning robot for spherical buildings, CLAWAR Newsletter 2002 p. 993 